\title{
On the Role of Coherent Structures in a Lid Driven Cavity Flow
}

\author{
Benjamin Kadoch, Emmanuel Leriche, Kai Schneider, and Marie Farge
}

\begin{abstract}
The coherent vortex extraction (CVE) is a technique based on the nonlinear filtering of the vorticity field projected onto an orthonormal wavelet basis. The coherent vortices of the flow are reconstructed from few strong wavelet coefficients, while the incoherent background flow corresponds to the majority of weak wavelet coefficients. Here CVE is applied to a lid driven cavity flow. Only $2.3 \%$ of wavelet coefficients are necessary to capture the coherent structures and contains almost all the enstrophy. The incoherent flow, which is the remaining, is structureless and noise-like. The results show that lid driven cavity flows are characterized by the presence of pronounced coherent structures.
\end{abstract}

\section{Introduction}

This project aims to investigate the coherent structures appearing in the so-called Lid Driven Cavity (LDC) flow. The LDC consists of a 3D cubic fully confined

Benjamin Kadoch

M2P2-UMR 6181 CNRS \& CMI, Universités d'Aix-Marseille, 39, rue F. Joliot- Curie, 13453 Marseille Cedex 13, France

e-mail: kadoch@L3m.univ-mrs.fr

Emmanuel Leriche

Faculté des Sciences et Techniques, Université Jean-Monnet, rue du Docteur Paul Michelon 23, Saint-Étienne, F-42023, France

e-mail:emmanuel. leriche@univ-st-etienne.fr

Kai Schneider

M2P2-UMR 6181 CNRS \& CMI, Universités d'Aix-Marseille, 39, rue F. Joliot- Curie, 13453 Marseille Cedex 13, France

e-mail: kschneidecmi.univ-mrs.fr

Marie Farge

LMD-CNRS, ENS, 24 rue Lhomond, 75231 Paris Cedex 5, France

e-mail: farge@lmd.ens.fr 
domain, where the flow is generated by the motion of one of the wall's cavity, see the flow domain and naming conventions given in Fig. 1 The fluid enclosed in the cavity is assumed to be incompressible, viscous, Newtonian and homogeneous. The LDC is an archetype for internal recirculation flows and a benchmark for methods of direct numerical simulations. By opposition with the two-dimensional LDC flow which has become a standard benchmark problem for testing the accuracy of new numerical methods, the three-dimensional lid-driven cavity flow has received much less attention in the literature [9]. Besides its theoretical interest, the lid-driven cavity is a simplification of several engineering situations such as slots on the walls of heat exchangers or coating devices used in the paper industry for producing high grade paper and photographic film. Even though the problem statement appears fairly simple, the flow phenomena encountered within such systems are many and poorly understood. From a physical point of view, several well known flow phenomena appear in the LDC like shear flow, boundary layers, eddies and core vortex, and, with the occurrence of curved streamlines, a contribution due to the associated (Görtler) instability mechanism to the dynamics of the flow, Taylor-Görtler-like vortices. Less well known aspects of this flow concern the presence of streaks near the moving wall, of jet impingement at the walls, and of corner spiraling vortices and the coexistence of adjacent regions of laminar and turbulent flow within the cavity [6, 7, 2, 5].

\section{Numerical Simulation and Coherent Vortex Extraction}

Direct numerical simulation (DNS) -which involves the solution of the transient, non-linear, three dimensional Navier-Stokes equations without any modeling, and thus provides a complete description of a turbulent flow in space and time (from the largest turbulence generating eddies, down to the smallest dissipative Kolmogorov scales) - is performed at Reynolds numbers (based on the maximum velocity on the lid, and the cavity width), between $1.210^{4}$ and $2.210^{4}$. An analysis based on the mean Navier-Stokes equation and the Reynolds stresses budgets is provided in [6, 7]. The DNS use a spectral method with up to 5 millions Chebyshev collocation points, which enable the detailed representation of all dynamically significant scales of motion. The agreement with the available experimental data of Prasad et al. [8] is fairly good but the comparison is limited to the mid-plane.

To study the role of coherent structures in such a flow, the coherent vortex extraction (CVE) is applied at a given time. The CVE method is based on denoising theory [3] and does not assume any pattern for the coherent structures. First, the wavelet coefficients of the vorticity $\boldsymbol{\omega}=\boldsymbol{\nabla} \times \boldsymbol{u}$ are computed using the fast wavelet transform. A thresholding is then applied to the wavelet coefficients $\tilde{\boldsymbol{\omega}}_{\lambda}$. Thus, we split the field into two contributions, the wavelet coefficients of the coherent part defined by

$$
\tilde{\boldsymbol{\omega}}_{\lambda_{C}}=\left\{\begin{array}{ccc}
\tilde{\boldsymbol{\omega}}_{\lambda} & \text { if } & \left|\tilde{\boldsymbol{\omega}}_{\lambda}\right|>\varepsilon=\sqrt{4 / 3 \mathrm{Z} \ln \mathrm{N}} \\
0 & & \text { else }
\end{array}\right.
$$




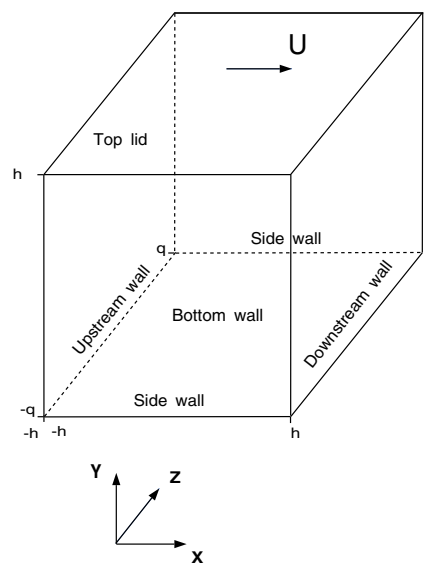

Fig. 1 Sketch of the flow domain $\Omega$

and those of the incoherent part as the remainder. The indices ${ }_{C}$ and ${ }_{{ }_{I}}$ denote the coherent and incoherent part, respectively, $Z=1 / 2\langle\boldsymbol{\omega}, \boldsymbol{\omega}\rangle$ is the enstrophy and $N$ the resolution. A first threshold is calculated from the total field then we apply the thresholding. The next step consists in splitting the total field with a threshold calculated from the incoherent field. Thus, this process is iterated with the threshold based on the new coherent field [1]. In this study this thresholding is applied with three iterations. The coherent $\boldsymbol{\omega}_{C}$ and incoherent $\boldsymbol{\omega}_{I}$ vorticity is reconstructed using the fast inverse wavelet transform. The two fields thus obtained are orthogonal by construction. As a consequence the separation of the total enstrophy is $Z=Z_{C}+Z_{I}$ and $\omega_{C}+\omega_{I}=\omega$.

\section{Results}

\subsection{Vorticity}

In the following we use the coherent vortex extraction to filter the vorticity field of a lid driven cavity flow corresponding to a Reynolds number $R e=1.210^{4}$. The DNS data computed with a Chebyshev collocation method on a non-equidistant grid with resolution $129^{3}$ has been reinterpolated on an equidistant grid with resolution $N=256^{3}$. Table 1 shows that almost all the enstrophy is retained in the coherent part, while the enstrophy of the incoherent part is negligible. The compression rate is hereby $2.26 \% \mathrm{~N}$, corresponding to the number of wavelet coefficients retained in the coherent part. The compression rate is slightly increased with respect to homogeneous isotropic turbulence at similar resolution $\sim 3 \%$ [4]. Moreover, the skewness and flatness of the total and coherent vorticity are similar. 
Table 1 Enstrophy $Z$, skewness, flatness and compression rate of the total, coherent and incoherent vorticity

\begin{tabular}{lccccc}
\hline & Enstrophy $Z$ & $\% Z$ & \multicolumn{2}{c}{ skewness flatness compression } \\
& & & & & rate \\
\hline total: & 3.59 & 100 & 32.2 & 2200 & $100 \%$ \\
coherent: & 3.59 & 99.99 & 32.2 & 2200 & $2.26 \%$ \\
incoherent: & $1.2810^{-5}$ & $3.610^{-4}$ & -0.0432 & 16 & $97.74 \%$ \\
\hline
\end{tabular}

In Fig. 2, the PDFs of total, coherent and incoherent vorticity are plotted. We remark that the total and coherent vorticity are nearly the same, while the incoherent part has a strongly reduced variance. As shown in the inset, the latter presents a shape close to an exponential behavior.

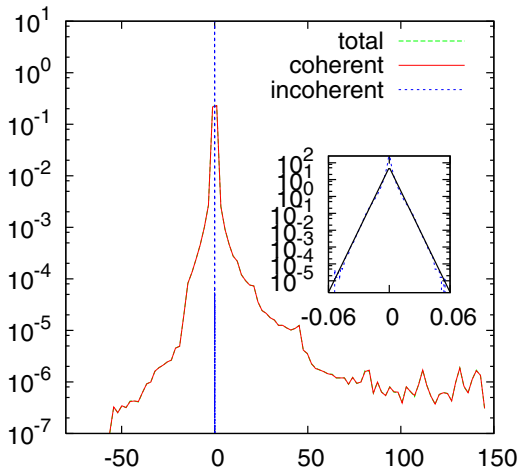

Fig. 2 PDFs of total, coherent and incoherent vorticity. Inset: PDF of incoherent vorticity

Figure 3 shows isosurfaces of vorticity modulus for the total, coherent and incoherent parts. The structures are retained in the coherent part. The incoherent part exhibits a structureless flow like a background noise, in almost all the domain except near the walls where some small sheets structures appear.

\subsection{Velocity}

To reconstruct the velocity fields, which are solenoidal, from the vorticity fields, the irrotational part has to be taken into account. Knowing that the velocity $\boldsymbol{u}$ can be decomposed into rotational and irrotational components (Helmholtz decomposition) $\boldsymbol{u}=\boldsymbol{u}_{s}+\boldsymbol{u}_{p}=\boldsymbol{\nabla} \times \boldsymbol{\psi}+\boldsymbol{\nabla} \phi$ where $\boldsymbol{\psi}$ is the potential vector and $\phi$ is the scalar potential which is harmonic $\left(\nabla^{2} \phi=0\right.$ by taking into account the boundary conditions, see fig. 1. Inverting the curl operator only allows to reconstruct its rotational component. To obtain the irrotational velocity we proceed as follows: 
(a) $|\omega|$

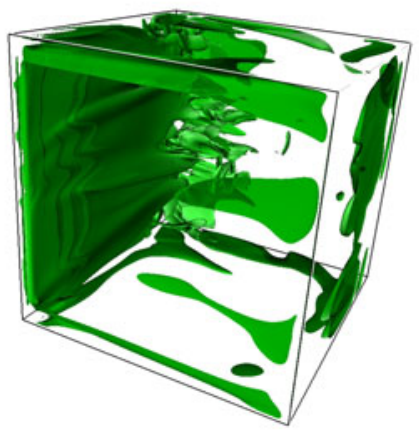

(b) $\left|\omega_{c}\right|$

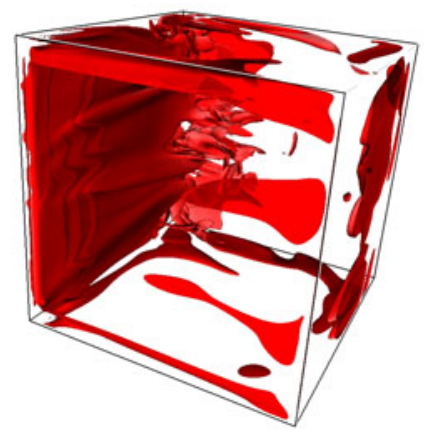

(c) $\left|\omega_{i}\right|$

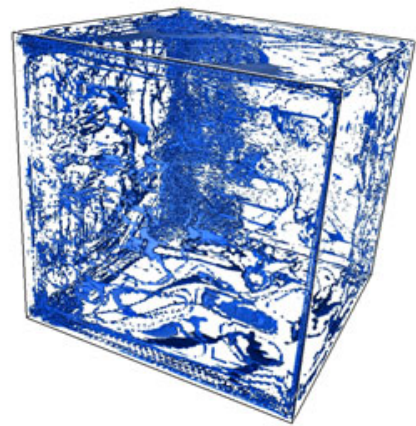

Fig. 3 Visualization of the modulus of the total, coherent and incoherent vorticities. The isosurfaces used are $10 / 9 \sigma^{2} \sim 2$ for the total and coherent part and $1 / 120 \sigma^{2} \sim 0.015$ for the incoherent part, where $\sigma^{2}$ is the variance of the total flow

- A Poisson equation is solved for each component of the potential vector imposing homogeneous Dirichlet boundary conditions, i.e. $\nabla^{2} \boldsymbol{\psi}=-\boldsymbol{\omega}$ with $\left.\boldsymbol{\psi}\right|_{\partial \Omega=0}$ and where $\nabla^{2}$ denotes the Laplace operator.

- The rotational velocity is computed using $\boldsymbol{u}_{s}=\boldsymbol{\nabla} \times \Delta^{-1} \boldsymbol{\omega}$. A Fourier spectral Poisson solver is used supposing the periodicity of the vorticity fields. Then the curl is applied to compute the velocity from $\boldsymbol{\psi}$.

- The irrotational velocity is deduced from the velocity: $\boldsymbol{u}_{p}=\boldsymbol{u}-\boldsymbol{u}_{s}$.

- The coherent rotational velocity is then $\boldsymbol{u}_{s}{ }^{c o h}=\boldsymbol{\nabla} \times \Delta^{-1} \boldsymbol{\omega}_{\text {coh }}$.

- The coherent velocity is the sum of the coherent rotational velocity and the irrotational velocity $\boldsymbol{u}^{c o h}=\boldsymbol{u}_{s}{ }^{\text {coh }}+\boldsymbol{u}_{p}$.

- The incoherent velocity is equal to $\boldsymbol{u}^{i n c}=\boldsymbol{u}_{s}{ }^{i n c}$.

Almost all the energy of the flow is retained in the coherent contribution as illustrated in Table 2. The energy of the incoherent part could be also neglected, like for the incoherent enstrophy. 
Table 2 Energy $E$, skewness, flatness and compression rate of the total, coherent and incoherent velocity

\begin{tabular}{|c|c|c|c|c|}
\hline & Energy $E$ & $\% E$ & skewness & flatness \\
\hline total: & $7.7310^{-3}$ & 100 & 2.83 & 58.4 \\
\hline coherent: & $7.7310^{-3}$ & 100 & 2.83 & 58.4 \\
\hline incoherent: & $3.4910^{-9}$ & $4.1510^{-5}$ & $-0.1810^{-2}$ & 5.58 \\
\hline
\end{tabular}

The PDFs of the total, coherent and incoherent velocity are plotted in Fig. 4. Likewise the vorticity, the total and coherent velocity PDFs are fairly similar, and the PDF of incoherent part is close to an exponential and exhibits also a strong reduced variance.

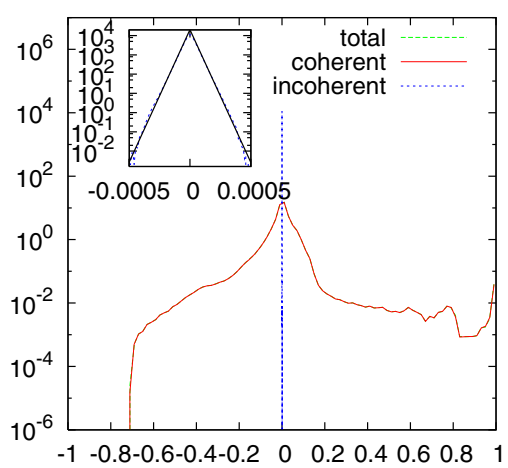

Fig. 4 PDFs of total, coherent and incoherent velocity. Inset: PDF of incoherent velocity
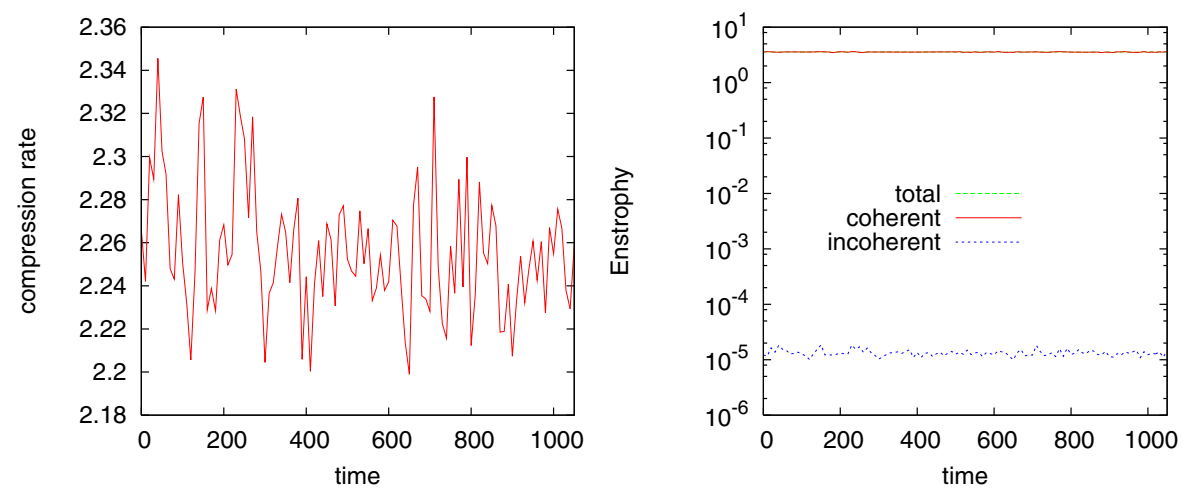

Fig. 5 Time evolution of compression rate (left) and time evolution of total, coherent and incoherent energy (right) 
Table 3 Mean and variance of enstrophy $Z$ and compression rate of the total, coherent and incoherent vorticity

\begin{tabular}{lccc}
\hline & Enstrophy $Z$ & $\% Z$ & $\begin{array}{c}\text { compression } \\
\text { rate }\end{array}$ \\
\hline mean & & & \\
\hline total: & 3.56 & 100 & $100 \%$ \\
coherent: & 3.56 & 99.99 & $2.26 \%$ \\
incoherent: & $1.3310^{-5}$ & $3.710^{-4}$ & $97.74 \%$ \\
\hline variance & \multicolumn{3}{c}{} \\
\hline total: & $1.210^{-3}$ & & 0 \\
coherent: & $1.210^{-3}$ & $8.9610^{-4} \%$ \\
incoherent: & $2.6810^{-12}$ & $8.9610^{-4} \%$ \\
\hline
\end{tabular}

\subsection{Application of CVE to the Flow Evolution}

To check the efficiency of CVE and the variability of the results for different time steps, we apply CVE to a set of 106 snapshots . Fig.5 5hows the time evolution of the compression rate and the time evolution of total, coherent and incoherent enstrophy contributions. The mean and variance values of compression rate and total, coherent and incoherent enstrophy are summarized in Table 3. It shows that the statistics are well converged and it confirms that the results and conclusions obtained in the previous section.

\section{Conclusion}

In conclusion, the coherent vortex extraction was applied to DNS data of a lid driven cavity flow. A detailled study is performed at one time. The total and coherent vorticities yield almost the same behavior, while the incoherent vorticity is negligible. As a consequence, the coherent structures are responsible for this kind of flow. Moreover the CVE is slightly more efficient than in homogeneous isotropic turbulence. Indeed the compression rate shows that less than $3 \%$ of wavelet coefficients are necessary to represent the coherent structures. These results motivate the use of Coherent Vortex Simulation (CVS) to model the time evolution of lid driven cavity flows.

Acknowledgements. This work was supported by the ANR project 'M2TFP', the DFG-CNRS Research Program 'LES and CVS of Complex Flows'. We thank Michael Schlegel from TU Berlin, for fruitful discussion on the reconstruction of the velocity field.

\section{References}

1. Azzalini, A., Farge, M., Schneider, K.: Nonlinear wavelet thresholding: A recursive method to determine the optimal denoising threshold. Appl. Comput. Harmon. Anal. 18, 177 (2005) 
2. Bouffanais, R., Deville, M.O., Leriche, E.: Large-Eddy Simulation of the flow in a LidDriven Cubical Cavity. Physics of Fluids 19(5), 055108 (2007)

3. Donoho, D., Johnstone, I.: Ideal spatial adaptation via wavelet shrink-age. Biometrika 81, 425 (1994)

4. Farge, M., Pellegrino, G., Schneider, K.: Coherent vortex extraction in 3D turbulent flows using orthogonal wavelets. Phys. Rev. Lett. 97, 054501 (2001)

5. Habisreutinger, M.A., Bouffanais, R., Leriche, E., Deville, M.O.: A Coupled Approximate Deconvolution and Dynamic Mixed Scale Model for Large-Eddy Simulation. Journal of Computational Physics 224(1), 241-266 (2007)

6. Leriche, E., Gavrilakis, S.: Direct Numerical Simulation of the Flow in a Lid-Driven Cubical Cavity. Physics of Fluids 12(6), 1363-1376 (2000)

7. Leriche, E.: Direct Numerical Simulation in a Lid-Driven Cubical Cavity at High Reynolds Number by a Chebyshev Spectral Method. Journal of Scientific Computing 27(1-3), 335-345 (2006)

8. Prasad, A.K., Koseff, J.R.: Reynolds number and end-wall effects on a lid-driven cavity flow. Physics of Fluids 1(2), 208-218 (1989)

9. Shankar, P.N., Deshpande, M.D.: Fluid Mechanics in the driven cavity. Annu. Rev. Fluid Mech. 2000 32, 93-136 (2000) 